\title{
A NOTE ON THE MOST "DANGEROLS" AND SKEWEST CLASS OF DISTRIBUTIONS
}

\author{
Gunnar Benktander \\ Solna, Sweden
}

In the classical definition skewness is departure from symmetry. It was therefore natural to measure skewness by using a normalized third moment $\mu_{3} / \sigma_{3}$. This condensed measure, however, is not refined enough to be used as an operational instrument for studying various functions which might be used to describe actual claim distributions. This is true especially when the interest is concentrated towards the higher values of the variate.

In their paper (I) Benktander-Segerdahl have suggested that the average excess claim $m(x)$ as a function of the priority $x$ should be used to reveal the characteristics of the tail of the distribution

$$
m(x)=\frac{\int_{x}(z-x) d P(z)}{\mathrm{I}-P(x)}=\frac{\int_{x} H(z) d z}{H(x)}
$$

where $P(x)=\mathrm{I}-H(x)$ denotes the distribution function.

This statistic is very apt when comparing actual claim distributions with possible theoretical models. It is also useful when classifying these models.

If, however, emphasis mainly is laid on classifying distributions according to their skewness, another statistic might be preferable. Let $\mu(x) d x$ denote the probability that a stochastic variable which is known to be at least equal to $x$, does not exceed $x+d x$. In other words, $\mu(x) d x$ represents the probability that a claim or the corresponding stochastic variable, which, when observed from the bottom, is "alive" at $x$, "dies" in the interval $(x, x+d x)$. The lower this claims rate of mortality, the skewer and more dangerous is the claim distribution. 
We obviously have

$$
\mu(x) d x=\frac{d P(x)}{\mathrm{I}-P(x)}=-\frac{d H(x)}{H(x)}
$$

or $\quad \mu(x) d x=-d \log H(x)$,

which gives $\log H(x)=\log c-\int^{x} \mu(t) d t$

$$
\begin{aligned}
& \text { and } H(x)=C e^{-\int^{-} \mu(t) d t} \\
& \text { or } \quad H(x)=e^{-\int_{a}^{x} \mu(t) d t} \text { if all } x \geqslant a .
\end{aligned}
$$

We shall show that

$$
\mu(x)=\frac{I+m^{\prime}(x)}{m(x)} .
$$

$m(x)=\frac{\int_{x} H(z) d z}{H(x)}$ can be written $H(x) m(x)=\int_{x} H(z) d z$.

Differentiation gives $H(x) m^{\prime}(x)+H^{\prime}(x) m(x)=-H(x)$

$$
\text { or } \frac{\mathrm{I}+m^{\prime}(x)}{m(x)}=-\frac{H^{\prime}(x)}{H(x)}=\mu(x)
$$

Supposing the variable $x$ is unlimited, we have $H(x)>0$ for all finite $x$. As $H(\infty)=0$, this implies that the integral

$$
\int_{x}^{\infty} \mu(t) d t \quad \text { must diverge. }
$$

If $\mu(x)$ is represented in the form $\mu(x)=k x p$, this condition is satisfied for all $p \geqslant-\mathrm{I}$. When $p>-\mathrm{I}$, the corresponding distributions are of exponential type. For $p=0$ we obtain the ordinary exponential distribution.

$p=-\mathrm{I}$ defines the Pareto class of distributions, because there

$$
H(x)=C e^{-\int^{x} \frac{k d t}{t}}=C x^{k}
$$

Within the given class of distributions those corresponding to $p=-$ I represent a limit class characterized by the lowest possible "mortality" of the claims. Within the given frame the Pareto class thus represents a maximum of skewness in accordance with the definition of the notion given above. 
It might happen that distributions which are skewer than the Pareto distribution, exist. In order to detect them it is necessary to go outside the class defined by $\mu(x)=k x^{p}$.

If for example $\mu(x)=k_{1} x^{p_{1}}+k_{2} x^{p_{2}}+k_{3} x^{p_{3}}+\ldots \ldots$

$$
\begin{aligned}
& \text { where } p_{1}>p_{2}>p_{3}>\ldots \ldots \\
& \text { and all } k_{i}>0 .
\end{aligned}
$$

it is easy to see that the behaviour of the frequency function in the limit is governed by $p_{1}$.

$$
\text { If } \mu(x)=a+k x^{\mathbf{1}} \text { then } H(x)=C x^{k} e^{-a x} \text {. }
$$

For high values of $x$ the general behaviour of this function is governed by the exponential term.

If $\mu(x)=k_{1} x^{-1}+k_{2} x^{-2}$

then $H(x)=C x^{k 1} e^{-\frac{k_{2}}{x}}$,

which function obviously quickly converges towards the ordinary Pareto distribution.

Let us thus denote as the Pareto class in a wide sense the distributions defined by $\mu(x)$-expressions such that $\lim x \mu(x)=k$.

Let us study $\mu(x)=\frac{k}{x \log x}$.

The integral $\int_{x}^{\infty} \mu(t) d t$ diverges

and for $x>x_{o}$ the claims obviously have a lower "mortality" than when $\mu(x)=\frac{k}{x}$.

We have $H(x)=C e^{-\int_{t \log t}^{x} t}=C e^{-\int_{t}^{x} \log \log t}$

$$
\text { or } \quad H(x)=C \frac{\mathrm{I}}{(\log x)^{k}} \text {. }
$$

This claim distribution does not possess a finite mean and is therefore-we hope-uninteresting for practical insurance purposes. 
The same observation is valid for distributions defined by

$$
\mu(x)=\frac{k}{x(\log x)^{q}} \text {, where } 0<q<\mathbf{r} .
$$

If we impose, which is natural, the condition of a finite mean, however, already part of the Pareto class will be cut out as impossible, namely those defined by $k \leqslant \mathrm{I}$. Distributions defined by a lower mortality rate than the Pareto class thus, intuitively-cannot possess finite means.

A strict proof of this has been given by Segerdahl, who has shown that

$$
\mu(x)=\frac{\varepsilon(x)}{x}, \text { where } \lim _{x \rightarrow \infty} \varepsilon(x)=0
$$

defines a distribution with an infinite mean.

Thus, at least for distributions defined by $\mu(x)$ functions which for $x>x_{0}$ are monotonous or majored by monotonous functions, i.e. making exception only for pathological cases, we can state the following:

"The Pareto class in the wide sense is a limit class dividing those frequency functions for which all moments exist from those for which all moments diverge".

\section{REFERENCES}

(I) G. Benktander and C. -O. Segerdahl: "On the Analytical Representation of Claim Distributions with Special Reference to Excess of Loss Reinsurance", XVIth International Congress of Actuaries, Brussels, I960.

(2) L. G. Benckert and I. Sternberg: "An Attempt to find an Expression for the Distribution of Fire Damage Amount", XVth International Congress of Actuaries, New York, I957.

(3) E. J. Gumber: "Statistics of Extremes", Columbia University Press, New York I958. 